\title{
Acoustic Sensors to Measure Speed of Oil Flow in Downhole Pipes
}

\author{
$1^{\text {st }}$ Nafiseh Vahabi \\ Department of Electronic \\ and Electrical Engineering \\ University College London (UCL) \\ London, UK \\ Nafiseh.Vahabi.14@ucl.ac.uk
}

\author{
$2^{\text {nd }}$ Mohammed Alabdullah \\ Soudi Aramco \\ Dhahran, Saudi Arabia \\ mohammed.alabdullah@aramco.com
}

\author{
$3^{\text {rd }}$ David R. Selviah \\ Department of Electronic \\ and Electrical Engineering \\ University College London (UCL) \\ London, UK \\ d.selviah@ucl.ac.uk
}

\begin{abstract}
This study was conducted to estimate the downhole speed of flow in oil wells and determined the flow direction by analyzing acoustic data recorded by fibre optic distributed acoustic sensors. The signals generated from acoustic data are in the time versus distance domain that are then normalized and differentiated with respect to distance. A 2D Fast Fourier Transform is used to convert time to frequency and distance to wave-number for subsequent calculation. A Gamma correction function was employed to enhance an intensity of the signal in the frequency wevenumber domain. Also, decaying function was successfully applied to enhance the signals with a very low frequencies. We developed a novel method called integration along the radius in polar coordinate to measure the speed of sound and calculating the speed of oil flow. We compared the performance of our method with a Radon transform and proved our method outperforms an existing methods in both processing time and accuracy. The data sets used in this study are recorded from real oil and gas pipes which means there is no controlled environment and there are lots of noisy signals as a result of unpredicted events under the sea. The result of this study is applicable in Oil and Gas production energy industry, Hydraulic fracturing and shale gas extraction energy industry, Borehole water supply industry, Gas pipeline transportation energy industry and Carbon Dioxide Sequestration industry.

Index Terms-Flow speed measurement, normalization, line detection, radon transform, Fast Fourier Transform
\end{abstract}

\section{INTRODUCTION}

Downhole flow speed measurements have a great economic value for the oil and gas industry such as monitoring the flow speed at each point down a well in order to maximize oil and gas production and optimize the quality of oil and gas produced, whilst reducing the cost [1], [2]. Numerous approaches for flow speed measurements have been identified and developed which are classified into two groups: on-line and off-line techniques [3]. The on-line techniques are usually more complicated and can be done by use of sensors such as distributed optical fiber sensors, Distributed Temperature Sensor (DTS) [4], Electrical capacitance tomography (ECT) [5], kinematic wave detection [6] and ultrasonic measurements [7], [8]. The idea of these techniques is to develop an arrangement of instruments which provide localized measurements of pressure and temperature. One of the disadvantages of these technologies is that they are only able to measure the flow at specific positions along the down hole pipe and the instruments have to be installed prior to the pipe insertion [9]. Also, traditional techniques cannot monitor the wells in real time continuously. In addition, developing an algorithm for real time data acquisition needs to be high speed [10], which is difficult to achieve by tomographic techniques as they require complex image reconstruction [5]. Recent developments in distributed fiber technology use distributed pressure and temperature information to estimate the flow speed. However, the relationship between flow speed, pressure and temperature is uncertain and it is difficult to calculate the results accurately [7].

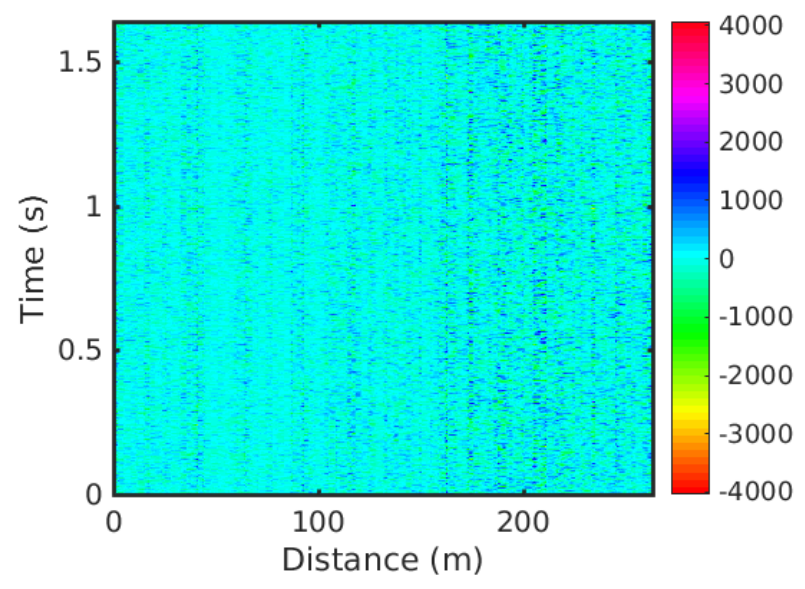

Fig. 1. Sample of raw acoustic data in distance (horizantal axis) and time (vertical axis).

The intelligent Distributed Acoustic Sensor (iDAS) developed by Silixa [11] is an optoelectronic system which records the true acoustic signal continuously along the path of sensing fiber tens of kilometers long [12]. When a pulse of light travels down an optical fiber, a small amount of the light is naturally backscattered and returns to the sensor unit. The nature of this scattered light is affected by tiny strain events within the optical fiber structure which themselves are determined by the localized acoustic signal. By recording the returning signal against time, a measurement of the acoustic field all along the fiber can be determined [13]. The iDAS has a frequency 
range from millihertz to hundreds of kilohertz. The optical fiber cables were permanently clamped and installed along the production/ injection tubing pipe. As the fluid mixture travels through the pipe its motion is very turbulent and it generates a noisy sound. The pipe acts like an acoustic wave guide that guides sound waves up and down the pipe. The sound is recorded continuously at every meter and this results in very large amounts of data being recorded daily, typically 48 Terabytes.

In this study, the raw data is pre-processed by applying normalization and filtering methods. The speed of sound is estimated by implementing a novel method which is summation along radius in polar coordinate and also Radon transform method. Having measured the speed of sound, we will consider the Doppler Effect [14] and calculate the flow velocity. The details of each technique are presented in section IV. A summary of the main findings are reported in the section $\mathrm{V}$, which compares the speed of flow versus depth calculated by each method.

\section{EXPERIMENTAL DATA}

Acoustic data is collected using the iDAS in an oil producer. The fibre is attached to the motherbore for up to $3427.3 \mathrm{~m}$ length. A sample of raw acoustic signals are presented in Fig. 1. The horizontal axis indicates the distance in metres with a spatial resolution of $0.51 \mathrm{~m}$. The vertical axis shows the stream of data for one second with a sampling rate of $10 \mathrm{kHz}$.

\section{Pre Processing Acoustic Data}

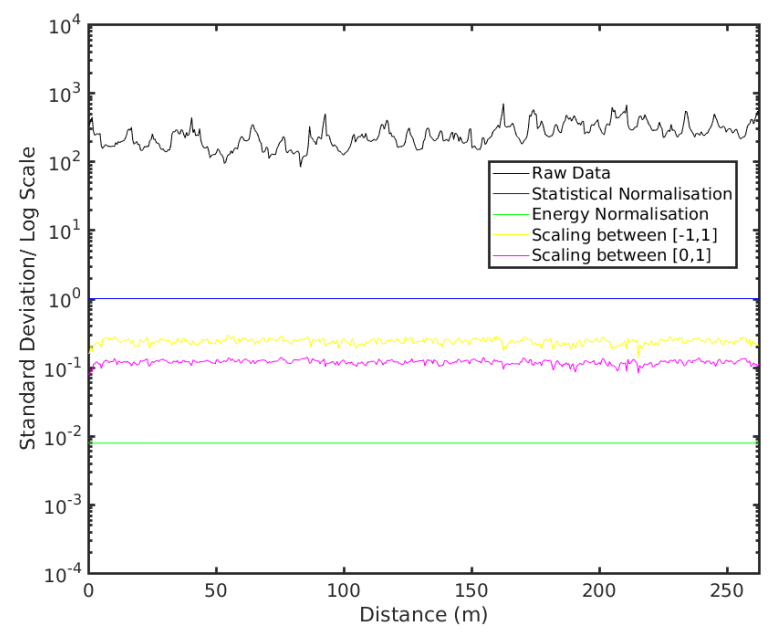

Fig. 2. Comparison of different normalization technique.

\section{A. Normalization}

Some parts of the optical fiber (iDAS) are tightly bound to the pipe and they record a higher amplitude acoustic signal, therefore it is advantageous to first perform a normalization. We implemented four normalization methods and compare the result to select the most suitable one for our dataset.
1) Statistical Normalization: Statistical or Z-score normalization uses the mean of signal, $\mu$, and its standard deviation, $\sigma$. The input signal is represented by a vector $x$, and its normalized version is given by $x^{\prime}$ :

$$
x^{\prime}=\frac{x-\mu}{\sigma}
$$

The Statistical Normalization reduces the effect of noise and outliers [15].

2) Energy Normalization: Energy Normalization divides the input signal by its energy norm:

$$
x^{\prime}=\frac{x}{\sqrt{x \cdot x}}
$$

where $x^{\prime}$, and $x$ are the output and input signals respectively.

3) Scaling: Scaling is sometimes referred to as min-max normalization in which the output is constrained between certain values. Most commonly, the input is rescaled to the range of [-1 1] or [0 1]. We scaled the acoustic signals between $\left[\begin{array}{ll}-1 & 1\end{array}\right]$ and [0 1] by using Eq. 3 and Eq. 4 respectively where $x^{\prime}$ is output signal and $x$ is input signal:

$$
\begin{gathered}
x^{\prime}=\frac{x-\mu}{\max (x-\mu)} \\
x^{\prime}=\frac{x-\min (x)}{\max [x-\min (x)]}
\end{gathered}
$$

Scaling uses linear mappings that does not affect the underlying distribution of data, thus keeping the same features [15].

To compare different normalization methods, standard deviation is calculated for each case. Fig. 2 shows the results of normalization in a logarithmic scale. Statistical normalization presents more consistent performance compared to the other methods.

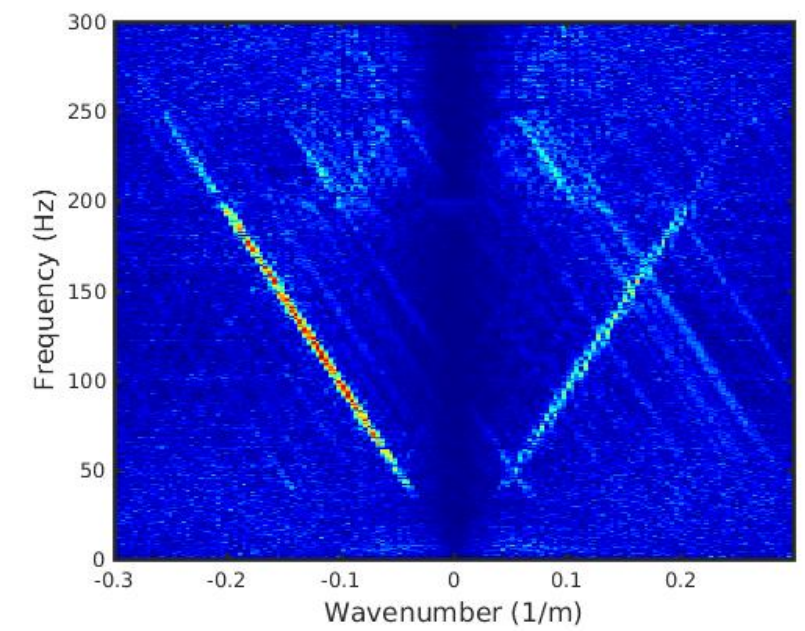

Fig. 3. Sample of acoustic data after applying 2D-FFT. The vertical axis shows frequency $(\mathrm{Hz})$ and the horizontal axis shows wavenumber $(1 / m)$ 


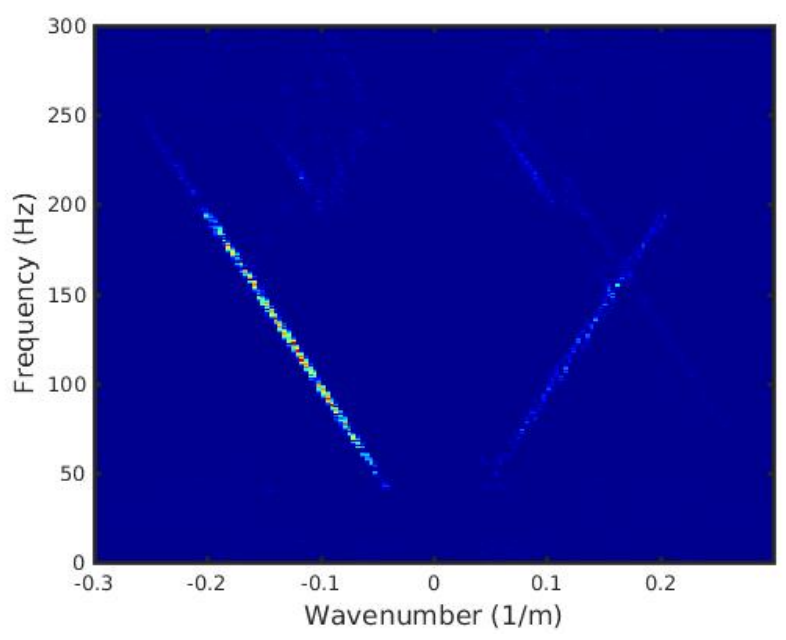

Fig. 4. The result of gamma correction on the V-shape image. The visibility of the V-shape enhanced after applying gamma correction function.

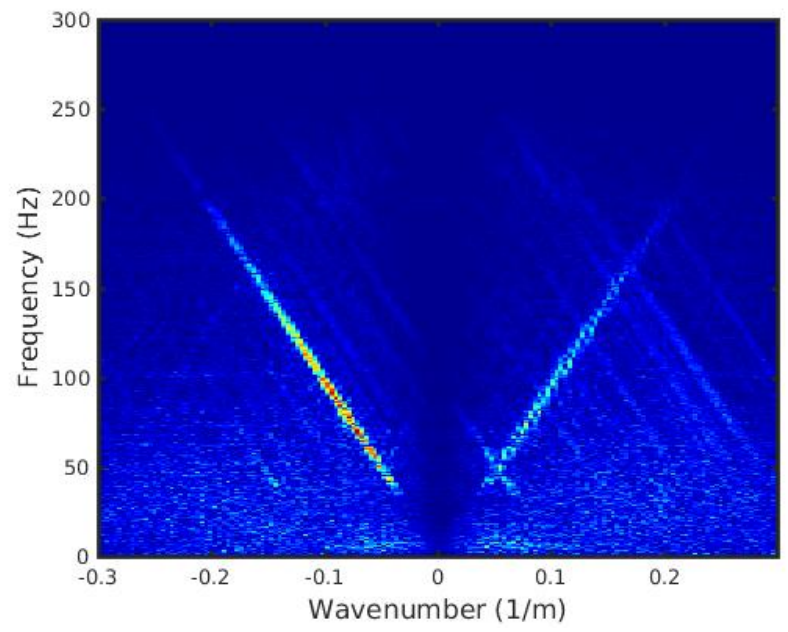

Fig. 5. The result of decaying function. The visibility of the V-shape enhanced after applying decaying function on the low frequency range.

\section{B. Filtering and Transformation}

As the data comes from real subsea wells a lot of sound external to the fluid filled pipe can be received by the optical fibre DAS. This may come from the sound of the waves on the sea surface, from submarines, whales, moving rocks on the sea floor. However, probably the strongest source of sounds is from adjacent wells. As all of these sources of sound are more than several pipe diamters away and certainly many acoustic wavelengths away, by the time the sound from any point source reaches the optical fibre DAS the phase front of the sound will have changed from spherical to almost a plane wave, so will impinge on the various parts and the effective microphones in the optical fibre DAS at the same time. Whereas sounds from the fluid flow itself, as it is so close to the optical fibre DAS, will still have spherical wavefronts from point sources. This means that such wavefronts will impinge on one effective microphone before they later impinge on another effective microphone. So we can remove the external sounds and keep the internal sounds by subtracting the sound waveform from one effective microphone from that from the adjacent effective microphone. This is the same as differentiating the dataset with respect to distance or depth.

2D Fast Fourier Transform (2D-FFT) is applied to convert data from time-distance domain to frequency-wavenumber domain. 2D-FFT can be computed efficiently where the dimensions of the samples are of power 2 [17]. We selected $2^{9}$ samples in distance and $2^{14}$ samples in time to maintain the Nyquist criterion [18]. Fig. 3 shows the acoustic data after applying 2D-FFT. The vertical axis corresponds to the frequency, whereas the horizontal axis corresponds to the wavenumber. The lines are corresponding to the speed of sound in the well have a V-shape (Fig. 3). The line with a positive gradient corresponds to the speed of sound in an upward direction and the line with a negative gradient corresponds to the speed of sound in a downward direction.

We can not achieve a clear V-shape within the full frequency range, therefore we apply an intensity transformation functions on the result of 2D-FFT to enhance the visibility of the V-shape. Gamma correction and decaying function are two operations which have been selected [17]. Gamma correction stretches the intensity of data by using:

$$
I_{\text {out }}=I_{\text {in }}^{\gamma}
$$

where $I_{\text {out }}$ and $I_{\text {in }}$ correspond to the input and output images respectively. The gamma constant, $\gamma$, determines if the stretching is compressive or expansive [17]. Fig. 4 shows the result of applying the gamma function with $\gamma=2.5$.

Our experiment shows the $\mathrm{V}$-shape line have a higher intensity in the range of frequencies between $30 \mathrm{~Hz}$ and 250 $H z$. Therefore we apply a decaying function 6 on k-f diagram (Fig. 3) to emphasise the visibility of lower range frequencies. Where $k$ and $f$ are the wavenumber, and frequency respectively. The constants $\alpha$ and $\beta$ determine how fast the decaying is along the wavenumber or frequency axes.

$$
I_{\text {out }}(k, f)=I_{\text {in }}(k, f) \times \exp \left(-\alpha k^{2}-\beta f^{2}\right)
$$

The result of applying decaying function can be seen in Fig. 5 .

\section{Detecting The Line}

Different approaches can be applied to detect the lines corresponding to the speeds of sound in k-f graph (Fig. 3). We can employ filters such as Sobel, Prewitt and Canny [17]. Then a linear model can be used to best fit the resultant points. Another approach is to transform the diagram using a Radon transform, or Hough transform to extract the straght lines. Radon transform usually gives a better resolution at the cost of processing time [19]. In this section, we implement a method, which depends on the polar transformation of the diagram and compare it with another method based on the Radon transform. 


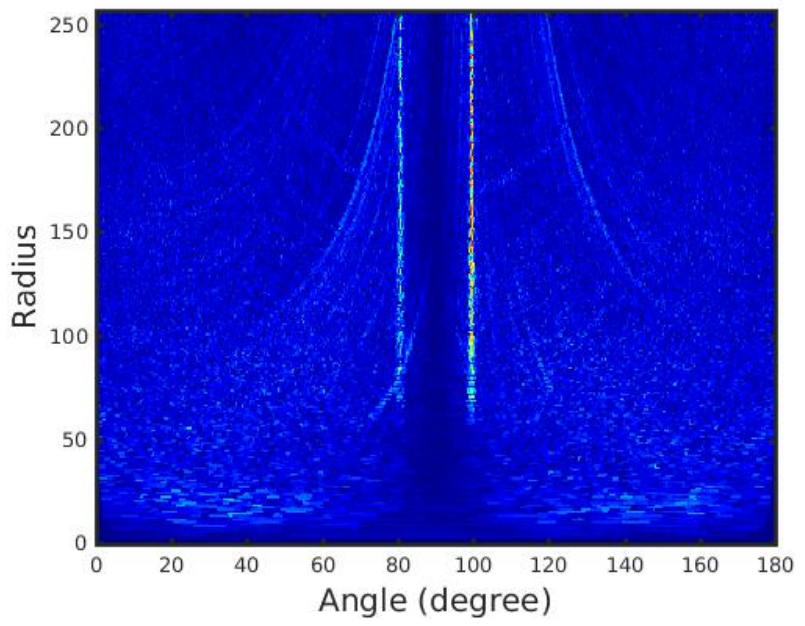

Fig. 6. Polar representation of acoustic data in frequecy wavenumber domain.

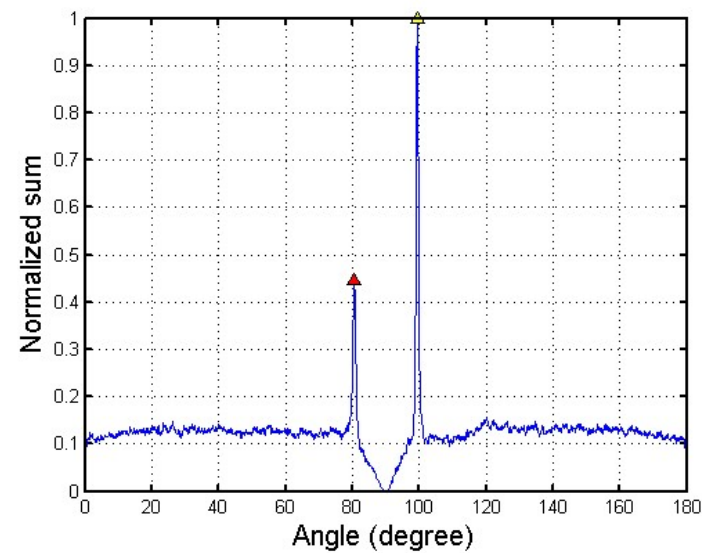

Fig. 7. Summation along the radius. Two peaks shows the location of the two lines.

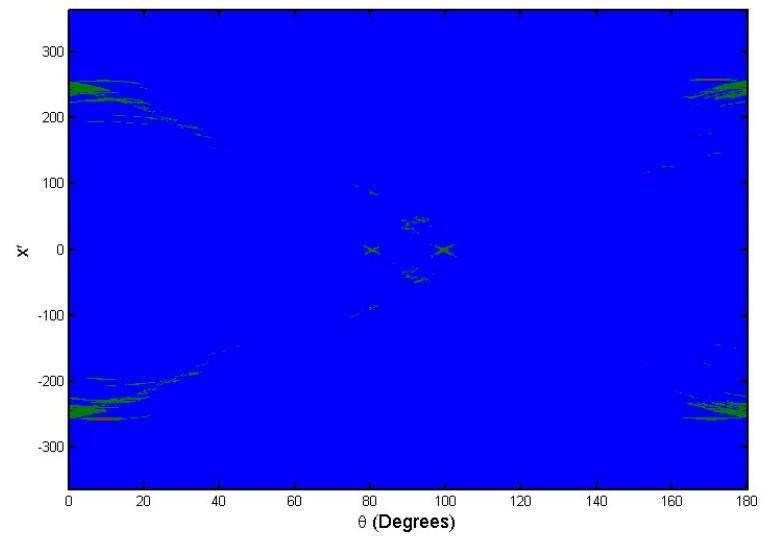

Fig. 8. The result of applying Radon transform on frequency wavenumber domain (positive and negative frequecy are considered).

\section{A. Integrating along the Radius in Polar Coordinates}

This method works by first applying the Polar transformation on the k-f diagram (Fig. 5) with the origin at $k_{0}=0$,

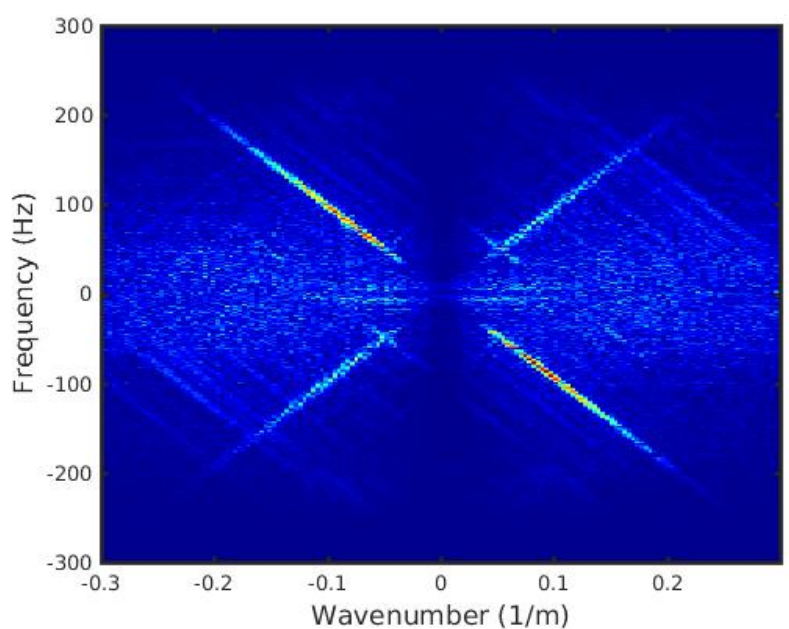

Fig. 9. Sample of acoustic data in frequency wavenumber domain with positive and negative frequency.

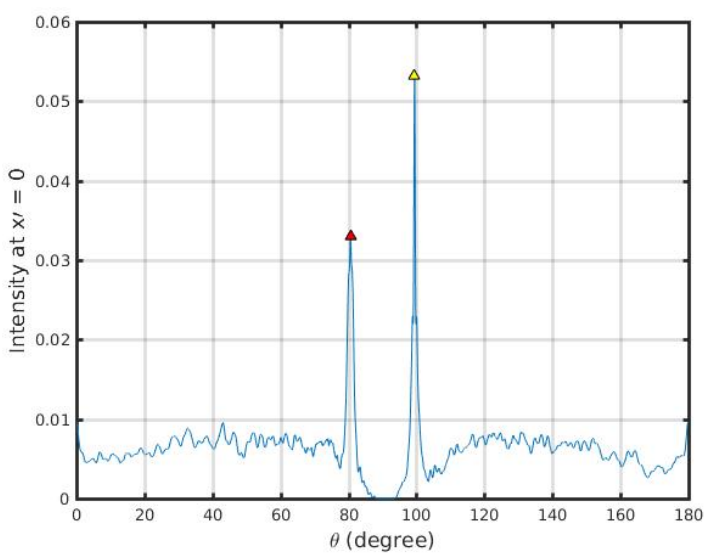

Fig. 10. Two peaks in radon transform.

and $f_{0}=0$. The basic relation to transform a function from cartesian coordinates to Polar coordinates is given by: $x=r \cos \theta$ and $y=r \sin \theta$. To simplify the mapping process with a discrete image, rounded values of the grid index are considered. A simplified form of the mapping is given by:

$$
I_{\text {out }}(m, n)=I_{\text {in }}\left(f_{0}+\operatorname{round}\left(r \sin \theta, k_{0}+\operatorname{round}(r \cos \theta)\right)\right.
$$

where $m$ and $n$ are coordinates of the polar graph corresponding to the radius and angle respectively. Their values are incremented as we increment $\theta$ and $r$. Values of $\theta$ are limited between $0^{\circ}$ and $180^{\circ}$. In addition, the maximum value of $r$ can have the smaller value of either the frequency length or half the wavenumber length. Resolutions of both $\theta$ and $r$ are specified carefully as a higher resolution would require an extra computational power but will improve accuracy of the result.

Fig. 6 represent the polar transformation of k-f digram. After applying the transformation, intensities are summed along the 
radius. The sum is normalised to have a maximum value of 1 . Fig. 7 shows the result of taking the sum along the radius. The peaks in the figure correspond to the lines in the k-f diagram. In order to detect the angles corresponding to the lines, the highest peaks need to be determined.

\section{B. Radon Transform}

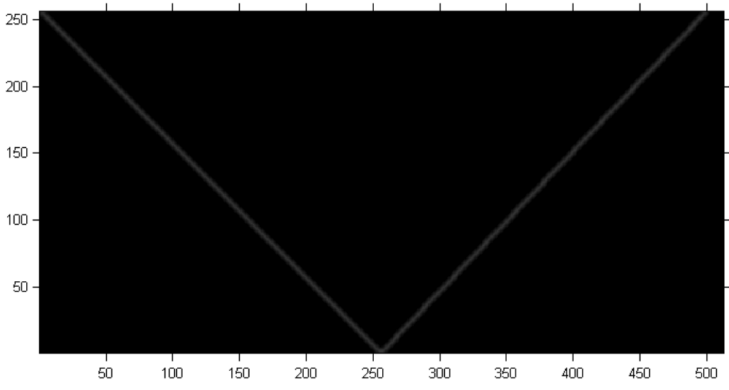

Fig. 11. Synthetic image.

The Radon transform is a projection of an image at specified directions. To apply the Radon transform on an image, intensities are summed along a set of predefined angles [16]. Radon transform of an image $f(x, y)$ is calculated by:

$$
g\left(x^{\prime}, \theta\right)=\int f\left(x^{\prime} \cos \theta-y^{\prime} \sin \theta, x^{\prime} \sin \theta+y^{\prime} \cos \theta\right) \mathrm{d} y^{\prime}
$$

where

$$
\left[\begin{array}{l}
x^{\prime} \\
y^{\prime}
\end{array}\right]=\left[\begin{array}{cc}
\cos \theta & \sin \theta \\
-\sin \theta & \cos \theta
\end{array}\right]\left[\begin{array}{l}
x \\
y
\end{array}\right]
$$

which means that $g\left(x^{\prime}, \theta\right)$ is a line integral of $f$ in a parallel direction to the $y^{\prime}$ axis. Before applying the Radon transform on the $k-f$ diagram the center of the image must correspond to $k=0$ and $f=0$. Fig. 8 shows the result of applying Radon transform, where the horizontal axis corresponds to the angle in degrees, and the vertical axis corresponds to the radial coordinates.

At $x^{\prime}=0$, an integral is taken at the centre of the diagram for each angle. So, we can determine the angles of lines by examining intensity values of the Radon transform at $x^{\prime}$ peaks of the resultant vector corresponds to the angles of the detected lines.

The peaks correspond to angles $80.5800^{\circ}$ and $99.4300^{\circ}$, compared to $80.5579^{\circ}$ and $99.4655^{\circ}$ which are obtained using the previous method.

\section{Result AND Discussion}

We implemented a novel method called integrating along the radius in polar coordinate to measure the speed of oil in downhole pipe. we compared the performance of our proposed algorithm with an existing method, Radon transform, using a synthetic image (Fig. 11), in which the angles of lines are known. We create a grey-level image $(256 \times 512)$, with two
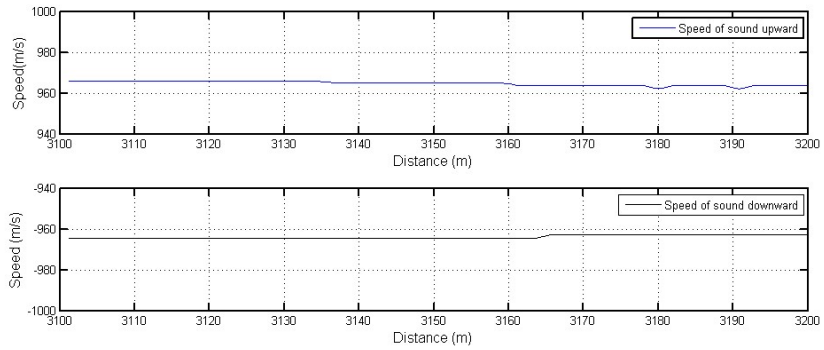

Fig. 12. Speed of sound along the wellbore.

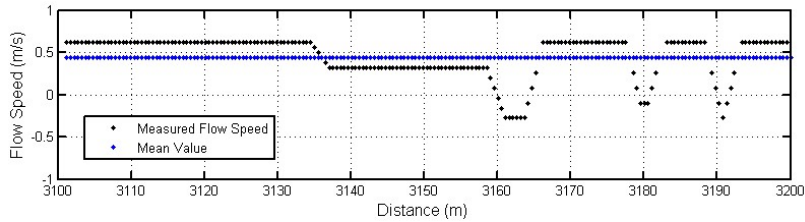

Fig. 13. Flow seed measurement along the wellbore.

lines. The lines are created such that they make angles of approximately $46.2628^{\circ}$ and $135^{\circ}$ with the horizontal axis.

The angle resolution in both methods can be specified and Table. I shows the comparison between the two methods for 3 different angle resolutions.

The error is measured as an absolute difference between the actual angle and the detected angle, divided by the actual angle. In this comparison, our method perfoms very well compared to the Radon method. Overall, the processing time of our method is less than Radon method and it is more accurate.

\section{A. Flow Speed Measurement}

Once the angles are detected, speeds of sound (upward and downward the wellbore) can be calculated using the following relation:

$$
\text { speed }=\tan (\theta) \frac{\Delta f}{\Delta k}
$$

where $\Delta(f)$ and $\Delta(k)$ correspond to the frequency and wavenumber resolutions respectively. The tangent of $\theta$ represents the slope of the line. For instance, angels $74.8856^{\circ}$, and $105.1378^{\circ}$ correspond to velocities $970.5836 \mathrm{~m} / \mathrm{s}$ and $-969.0154 \mathrm{~m} / \mathrm{s}$. This range of velocities is within the range of speed in crude oil. The process can be done 42 along the length of the wellbore, giving distributed measurements of speeds of sound as shown in Fig. 12.

A small change in the detected angle could signify a large change in the speed, partly because $\tan (\theta)$ is non-linear. Additionally, it is difficult to determine angles accurately in discretized images (rounded or interpolated values are taken). This can explains the sudden changes in Fig. 10.

Once the speeds of sound are obtained in both directions, the flow speed can be determined. The speed of sound travelling along the fluid flow will be faster due to the Doppler Effect [14]. The flow speed is given by: 
TABLE I

Comparing Two Line Detection Methods

\begin{tabular}{|c|c|c|c|c|}
\hline Method & Angle Resolution (degree) & Processing Time (s) & Detected Angle (degree) & Error $(\%)$ \\
\hline \multirow{3}{*}{ Sum along radius } & 0.01 & 0.1826 & $\begin{array}{c}46.4332 \\
135.2866\end{array}$ & $\begin{array}{l}0.3682 \\
0.2123\end{array}$ \\
\hline & 0.001 & 0.3615 & $\begin{array}{c}46.3037 \\
135.0143\end{array}$ & $\begin{array}{l}0.0885 \\
0.0106\end{array}$ \\
\hline & 0.0001 & 5.5494 & $\begin{array}{c}46.2734 \\
135.0043\end{array}$ & $\begin{array}{l}0.0230 \\
0.0032\end{array}$ \\
\hline \multirow{3}{*}{ Radon transform } & 0.01 & 9.1086 & $\begin{array}{c}45.3800 \\
134.0650\end{array}$ & $\begin{array}{l}1.9082 \\
0.6926\end{array}$ \\
\hline & 0.001 & 88.7680 & $\begin{array}{l}45.3810 \\
134.060\end{array}$ & $\begin{array}{l}1.9061 \\
0.6926\end{array}$ \\
\hline & 0.0001 & Operation aborted after 5 minutes & & \\
\hline
\end{tabular}

$$
\text { Flow Speed }=\frac{V_{u}-V_{d}}{2}
$$

where $V_{u}$ and $V_{d}$ are speeds of sound travelling upward and downward the pipe respectively. A negative result means that the fluid flows in a downward direction. The result of distributed flow measurements can be obtained along the wellbore as shown in Fig. 13.

Similar to the speeds of sound in Fig. 12, sudden changes occur in the measured flow speeds, as shown in Fig. 13 (obtained measurements are linearly dependent on the sound speeds). The average value of flow speed (in a $100 \mathrm{~m}$ interval) indicates that the flow is going upward at a rate of $0.41 \mathrm{~m} / \mathrm{s}$.

\section{CONCLUSION}

This paper successfully developed a novel methods to extract the flow velocity from the two dimensional acoustic data recorded by a fibre optic Distributed Acoustic Sensor in active Oil wells and also determined the direction of the flow. Our method demonstrated faster and more accurate estimation comparing with an existing method. The result of this study leads to direct industrial applications for real time condition monitoring of the behaviour of fluids along the whole length of fluid filled pipes.

\section{ACKNOWLEDGMENT}

The author, Nafiseh Vahabi, thanks Engineering and Physical Science Research council (EPSRC) for the funding studentship for her PhD. Also, the authors thank Soudi Aramco to provide the data and Silixa for using their sensors to record the data.

\section{REFERENCES}

[1] A., Chaudhuri, D. N., Sinha, A., Zalte, E., Pereyra, C., Webb, and Gonzalez, M. E., " Mass Fraction Measurements in Controlled OilWater Flows Using Noninvasive Ultrasonic Sensors," Journal of Fluids Engineering, vol. 3, 2014.

[2] M. J., Hill, A. D., Ehlig-Economides, C. and Zhu, D., "Petroleum production systems," Economides, Pearson Education, 2013.
[3] A., Chaudhuri, D. N, Sinha, A., Zalte, E., Pereyra, C., Webb and M. E., Gonzalez, "Distributed Acoustic Sensing a new tool for seismic applications," first break, vol. 32, pp.61-69, 2014.

[4] D. C., Finfer, V., Mahue, S., Shatalin, T., Parker and M., Farhadiroushan, "Borehole Flow Monitoring using a Non-intrusive Passive Distributed Acoustic Sensing (DAS)," Society of Petroleum Engineers, 2014.

[5] I. Ismail, J. Gamio, S. A. Bukhari, and W. Yang, "Tomography for multi-phase flow measurement in the oil industry," Flow Measurement and Instrumentation, vol. 16, pp. 145-155, 2005.

[6] G. P., Lucas and I. C. Walton, "Flow rate measurement by kinematic wave detection in vertically upward, bubbly two-phase flows," Flow Measurement and Instrumentation Journal, vol.8, pp.133-143, 1998.

[7] D. L., Gysling and M. R. Myers, "Distributed sound speed measurements for multiphase flow measurement", U.S. Patent 6,601,458, issued August $5,2003$.

[8] D. L., Gysling, "Fluid density measurement in pipes using acoustic pressures", U.S. Patent 6,971,259, issued December 6, 2005.

[9] C. Y., Chong and S. P., Kumar, "Sensor networks: evolution, opportunities, and challenges", Proceedings of the IEEE, vol. 37, no. 1, pp.12471256, 2003.

[10] A., Nehorai and E., Paldi, "Acoustic vector-sensor array processing,"IEEE Transactions on Signal Processing", vol. 42, no. 9, 24812491, 1994.

[11] Silixa's granted UK patents on the iDAS and flow monitoring methods. GB2482641, GB2517100 and GB251732 all Farhadiroushan, Parker and Shatalin.

[12] T. Parker, S. Shatalin and M., Farhadiroushan, "Distributed Acoustic Sensing a new tool for seismic applications", EAGE First Break, vol. 32 2014, 61-69.

[13] J. Xiao, M., Farhadiroushan, A., Clarke, R. A., Abdalmohsen, E., Alyan, T. R. Parkerand and H. C. Milne, "Intelligent Distributed Acoustic Sensing for In-well Monitoring," In SPE Saudi Arabia Section Technical Symposium and Exhibition, Society of Petroleum Engineers, 2014.

[14] V. C., Chen, F., Li, S. S., Ho and H., Wechsler, "Micro-Doppler effect in radar: phenomenon, model, and simulation study," IEEE Transactions on Aerospace and electronic systems, vol. 42, no. 1, pp. 2-21, 2006.

[15] K. L. Priddy and P. E. Keller, "Artificial neural networks: an introduction," SPIE Press, vol. 68, 2005.

[16] T., Olson, and J., DeStefano, "Wavelet localization of the Radon transform, "IEEE Transactions on Signal Processing, vol. 42, no. 8, pp.2055-2067, 1994.

[17] R. C. Gonzalez, R. E. Woods, and S. L. Eddins, "Digital image processing using MATLAB," Pearson Education India, vol. 634, 2004.

[18] R., Tao, Y. L., Li and Y., Wang, "Short-Time Fractional Fourier Transform and Its Applications, "IEEE Transactions on Signal Processing, vol. 58, no. 5, pp.2568-2580, 2010.

[19] M. van Ginkel, C. L. Hendriks, and L. J. van Vliet, "A short introduction to the Radon and Hough transforms and how they relate to each other, "The Quantitative Image Group Technical Report Series, 2004. 